\title{
Orale Antikoagulation
}

\section{Gerinnungsselbstmanagement erhöht die Sicherheit}

Bei der oralen Antikoagulation mit Vitamin-K-Antagonisten (VKA) ermöglicht das Gerinnungsselbstmanagement (GSM) eine engmaschige Qualitätskontrolle der Gerinnungseinstellung. Im Bedarfsfall ist eine sofortige Therapieanpassung möglich.

\section{Vitamin-K-Antagonisten weiterhin wichtige Säule der Antikoagulation}

Auch nach der Einführung der direkten oralen Antikoagulanzien (DOAK) bleiben VKA weiterhin ein wichtiger Therapiebestandteil. „Bei der Auswahl des geeigneten Antikoagulans sollten Risiko und möglicher Nutzen individuell für den und mit dem Patienten abgewogen werden“, betonte PD Dr. Jens Kreuz, Bad Kreuznach. Zu den Entscheidungskriterien zählen die Patientenkonstitution, die
Einschätzung der Blutungsneigung sowie Begleiterkrankungen und -medikationen. Während bei den VKA regelmäßig der International-Normalized-Ratio(INR)Wert zu überprüfen ist, wird bei DOAK derzeit kein Monitoring empfohlen.

\section{Experte in eigener Sache}

Angesichts der generell schlechten Einnahmetreue bei Langzeittherapien wie der oralen Antikoagulation kann die Kontrolle des Gerinnungswerts durch den geschulten Patienten einen wertvollen Beitrag zur Verbesserung der Therapieadhärenz leisten. Das GSM ermöglicht einen engmaschigen Überblick über die Qualität der Gerinnungseinstellung und eine sofortige Dosisanpassung. Zudem erhalte das GSM die Flexibilität und Unabhängigkeit des $\mathrm{Pa}$ - tienten im Alltag, führte Dr. Jens Heidrich, Hamburg, aus.

\section{Einfache Handhabung}

Das GSM gilt nach rund 25 Jahren Praxiserfahrung als leitliniengesicherter und kosteneffizienter Standard. Zur Verfügung steht zum Beispiel das einfach $\mathrm{zu}$ bedienende CoaguChek ${ }^{\circledR} \mathrm{XS}$ System, das sichere und mit dem Labor vergleichbare Messergebnisse liefert und eine schmerzarme Blutgewinnung ermöglicht. Für die Messung werden nur $8 \mu \mathrm{l}$ Blut aus der Fingerbeere benötigt.

\section{Gudrun Girrbach}

- Industriesymposium "Spannungsfeld Antikoagulation: Innovation versus Praxiserfahrung", 122. Kongresses der Deutschen Gesellschaft für Innere Medizin (DGIM); Mannheim, April 2016 (Veranstalter: Roche Diagnostics)

\section{Diabetes mellitus}

\section{Insulin aspart reduziert kardiovaskuläre Ereignisse}

_ Das eigentliche Ziel der Diabetestherapie ist die Verhinderung mikro- und makrovaskulärer Ereignisse. Während der günstige Einfluss auf mikrovaskuläre Folgeschäden als gesichert gilt, ist die Datenlage bezüglich makrovaskulärer Komplikationen wie Herzinfarkt und Schlaganfall deutlich dünner. Für Metformin konnte in der UKPD-Studie und für Empagliflozin in der EMPA-REGoutcome ${ }^{\varpi}$-Studie eine günstige Wirkung auf makrovaskuläre Komplikationen belegt werden. Auch für Liraglutid wurden in der LEADER-Studie positive Resultate angekündigt. Für Insuline gibt es bisher keine prospektiven kontrollierten Daten aus randomisierten Studien.

Doch eine retrospektive Datenbankanalyse in deutschen Hausarztpraxen ergab Hinweise, dass eine Therapie mit dem schnell wirksamen Insulinanalogon Insulin aspart (NovoRapid ${ }^{\circledR}$ ) im Vergleich zu Humaninsulin mit weniger makrovaskulären Ereignissen assoziiert ist [Rathmann W, Kostev K. Diab Obes Metabolism. 2012). Mit Insulin aspart konnten im Vergleich zu Humaninsulin die Inzidenz kardiovaskulärer Ereignisse um 26\%, die Myokardinfarktrate um $31 \%$, die Manifestation einer PAVK um $20 \%$ und das Auftreten eines Schlaganfalls/TIA um $42 \%$ reduziert werden.

Liebl et al. [Exp Clin Endolcrinol Diabetes 2014, doi: 10.1055/s-0034-1376987] haben hochgerechnet, was diese Ergebnisse ökonomisch bedeuten. Sie errechneten, dass acht Diabetiker drei Jahre lang mit Insulin aspart anstelle von

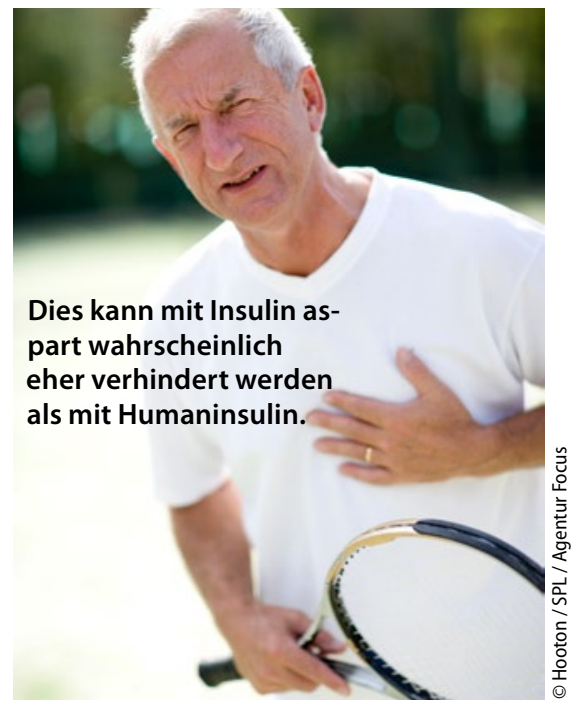

Humaninsulin behandelt werden müssten, um ein makrovaskuläres Ereignis zu verhindern.

Dr. Peter Stiefelhagen 